\title{
Selective amplification of salient features of visual memories during early memory consolidation
}

\author{
Chaipat Chunharas ${ }^{1,2^{*}}$, Timothy F Brady ${ }^{1}$, Vilayanur S Ramachandran ${ }^{1}$ \\ ${ }^{1}$ Department of Psychology, University of California, San Diego. \\ 9500 Gilman Drive, La Jolla, CA 92093-0109 \\ ${ }^{2}$ Department of Medicine, Faculty of Medicine, King Chulalongkorn Memorial Hospital, Chulalongkorn University \\ 1873 Rama IV Pathumwan Bangkok, Thailand, 10330 \\ *corresponding author, cchunharas@gmail.com
}




\begin{abstract}
When people need to remember a whole set of words or images, they tend to remember any particular item as more closely resembling the gist of the entire set than it really was. Here we show that when participants are asked to remember only a few items -- and so maintaining the distinctiveness of this item in memory is of particular importance -- memory for that item is distorted in the opposite direction, amplifying its salient features. In a sequence of 4 experiments, we asked participants to remember the aspect ratio and size of a rectangle and draw it after various delays. Participants reliably exaggerated its distinctive feature in every experiment. This distortion occurred not just at initial encoding but also during memory consolidation and persisted for several hours. Thus, when remembering only a few items, memory amplifies the distinctive features of these items, a form of adaptive memory distortion.
\end{abstract}

Keywords: Memory distortion | Consolidation | Visual Memory | Memory Biases 


\section{INTRODUCTION}

Due to limitations inherent in any biological system, our memories of recent events are not as vivid, authentic and reliable as they seem to us. Instead of recording every bit of information like a video camcorder, our memories are constructed - they are abbreviated, edited and retrieved in a manner consistent with pre-existing schemata (Bartlett, 1932). Such memory distortion is not random, but instead a property of how our memory system works (Schacter, Guerin, \& St. Jacques, 2011). One commonly found example of memory distortion is the systematic distortion of memory towards the gist information (Huttenlocher, Hedges, \& Duncan, 1991; Huttenlocher, Hedges, \& Vevea, 2000). Such distortions have been found in working memory, long-term memory, in the relationship between episodic and semantic memory, and for nearly all kinds of stimuli (Alvarez, 2011; Brady \& Alvarez, 2011; Dubé, Zhou, Kahana, \& Sekuler, 2014; Freyd \& Johnson, 1987; Hemmer \& Steyvers, 2009; Huang \& Sekuler, 2010; Huttenlocher et al., 1991, 2000; Spencer \& Hund, 2002). In all of these cases, when people need to remember the properties of an object and report them later, the responses tend to be shifted toward the prototype or the center of the relevant category. Computational models show that this helps us to minimize error, as a response toward the mean is less likely to be incorrect compared with a response away from the mean (Brady \& Alvarez, 2011; Huttenlocher et al., 1991, 2000), and this property of memory may also promote memory stability.

However, systematic biases toward the center of a category (i.e., attraction bias) are not always useful. For example, if we need to remember one particular object among other similar things (e.g. a particular dog at the dog park), attraction toward the center of the category would reduce distinctiveness and create more confusions. Under those circumstances, it may instead be optimal for memory to be biased away from the gist: maximizing the distinctive feature (i.e., contrast). This should result in memory holding a caricature version of that object (e.g., one more different from the center of the category than the actual item) instead of blending it together with the other similar objects. While attenuation of distinctive features (blending) promotes generalization, amplification of distinctive features may promote specificity. There is some evidence consistent with this idea, but in general, existing work supposes that that people's underlying representation of the stimulus features is unbiased and veridical. For example, previous work has shown that when presented with a caricature, people are more likely to recognize it and do so more quickly (Lee, Byatt, \& Rhodes, 2000; Mauro \& Kubovy, 1992). However, this is generally interpreted as showing that, while the underlying representation is accurate and uncaricatured, the matching process needed for recognition benefits from more extreme stimuli (which resemble other faces even less than a normal stimulus, giving a competitive advantage to the correct stimulus; e.g., Tanaka, 1996). Similarly, there is a significant literature on pattern separation in the hippocampus, particularly in the dentate gyrus; however, this literature largely assumes that only the cues needed to retrieve the object, not the actual object features, are made more distinctive (e.g., Yassa \& Stark, 2011). 
Thus it remains largely unknown whether the distinctive features of the object themselves are amplified or biased away from the category or prototype to enhance their distinctiveness in memory. To address this, we asked participants to remember simple objects with clear, distinctive features. We hypothesized that those features should be amplified during subsequent testing. To ensure that it was the representation itself that was affected and not the matching process, we use participants' drawing to measure their memory (a visual free recall). As our main test case, we ask about memory for a very simple stimulus: a rectangle. We hypothesized that for a rectangle, the intuitive comparison point is a square, and thus the aspect ratio between the 2 sides of the rectangle should become more pronounced in memory, i.e., participants should remember a more extremely rectangular rectangle. Our results showed evidence of this amplification of distinctive feature effect, as the ratio of memorized rectangles was larger after delays even when the rectangular ratios of the memoranda were initially larger than individual's prototypical rectangle. Interestingly, the effect was present even when a rectangle was the only item that subjects needed to remember. In follow-ups, we found that the amplification was larger at 7 minutes compared to 2 minutes delay - indicating that the amplification effect continues to strengthen with increasing delay. Thus, contrary to popular wisdom, memory formation involves more than silent incubation, memories are changed to make them more distinctive as they are consolidated, a process we term distinctiveness exaggeration or caricaturization of memories.

\section{EXPERIMENT I}

We first investigated whether the memory for the ratio of the sides of a rectangle was amplified by asking participants to remember an image with a rectangle and later draw it from memory. Since a significant previous literature suggests that rather than amplification we should expect the ratio of the drawn rectangle to become closer to the prototypical rectangle e.g. (Hemmer \& Steyvers, 2009; Huttenlocher et al., 2000) we first elicited participants' prototypical rectangle from them and we designed a one-trial recall task where the stimuli to be remembered were already more rectangular than each participants' own rectangle prototype. Thus, if the ratio of the sides of the rectangle is amplified even further, such amplification cannot be explained by attraction towards the prototypical rectangular ratio.

\section{Methods}

Participants. We based the effect size on a similar drawing task of the well-known boundary extension effect (minimum number of participants of 6 with previous reported Cohen's $\mathrm{d}$ of $\sim 1.8$, alpha $=0.05$, power $=0.80$ ) (Hubbard, Hutchison, \& Courtney, 2010; Intraub \& Richardson, 1989). Twelve healthy volunteers ( 7 female, mean age of $20.9 \pm 0.6$ S.E.M.) from the University of California San Diego (UCSD) community participated in the experiment. All procedures were approved by the UCSD Institutional Research Board. All participants reported normal or 
corrected-to-normal vision without color-blindness and provided written informed consent. Participants were naïve to the purpose of the study and received course credits for their time. Three participants failed to show up to both sessions and so did not produce data.

Stimuli. Since each person may have a different prototypical rectangular ratio, we first estimated each individual's prototypical rectangular ratio. To do so, we asked each participant to draw a rectangle -- the first rectangle that comes to mind. Then, we measured all four sides of drawn rectangles and computed the ratio by dividing the average length of two long sides by the average of two short sides. The computed ratios were used to make a new, exaggerated rectangle (1.2 times more rectangular than the ratio they drew) that each participant had to remember in the main experiment. To distract subjects from the main goal of the experiment, we included multiple bits of task-irrelevant information. Specifically, the memoranda consisted of two geometric shapes - a specific rectangle and a square (with the same total area). They had different colors and positions such that the lengths of the shapes could not be directly compared (Figure 1). Participants were instructed to remember all of this information.

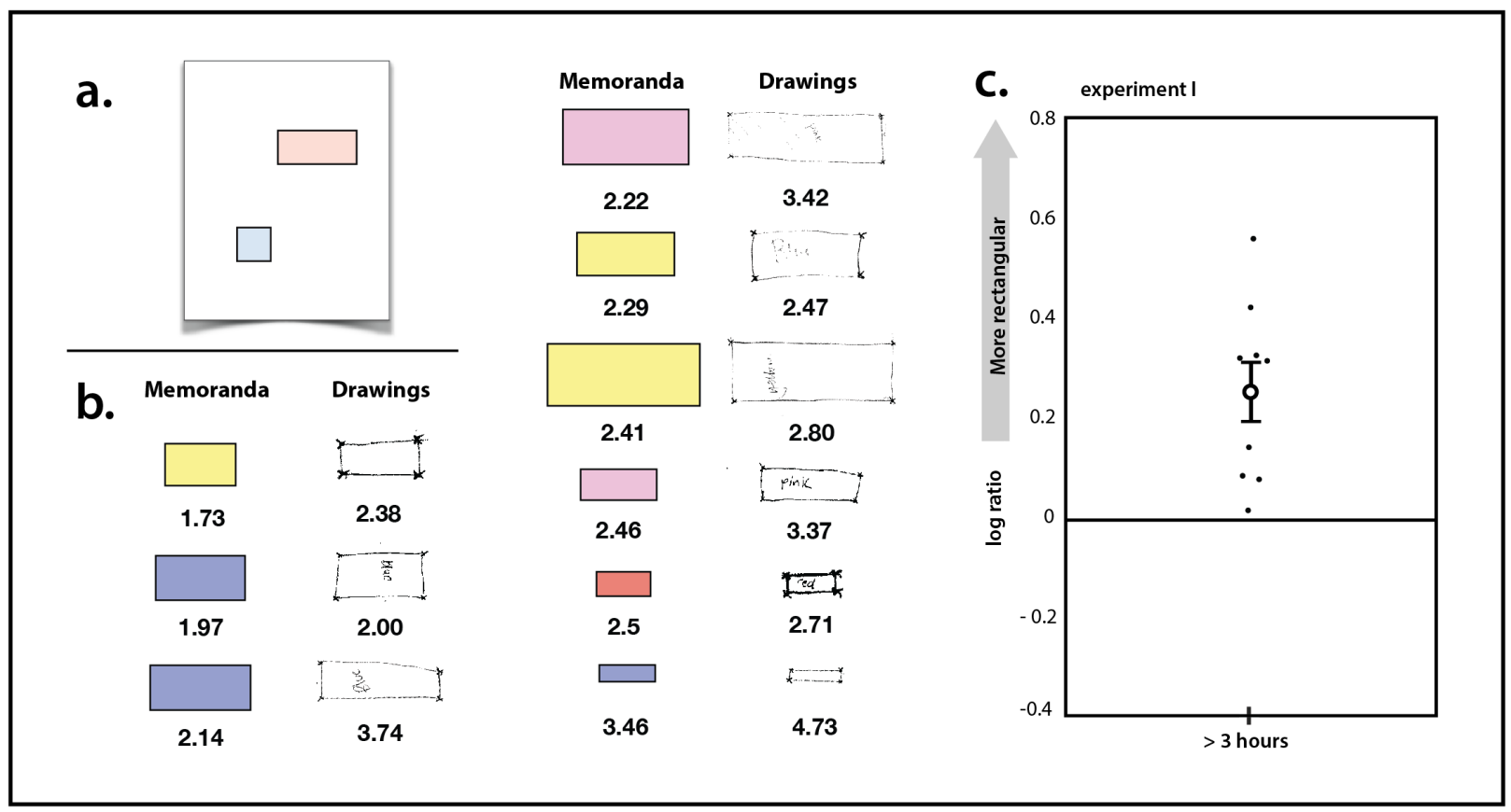

Figure 1. Data from Experiment 1. a.) Example of an image generated for a particular participant; Note that we included a square as a distractor. b.) Each pair shows the rectangle a given participant studied (in color) and then the rectangle drawn by that same participant when their memory was subsequently probed. The numbers are the ratios of the width to the height. All subjects drew a more amplified rectangle after $>3$ hours delay. c) The degree of amplification as quantified by the log ratio of the response drawing ratio vs. study item ratio was significantly higher than 0 . The error bar is $+/-1$ standard errors of the mean (S.E.M.) and each dot represents the data from one participant. 
Procedure. Two weeks after subjects initially drew their prototypical rectangle, we gave them a specific memorandum. This was specifically created for each person and instructed them to "remember every detail on the paper as precisely as you can". After a 3 hour delay, each participant received a blank sheet of paper and was instructed to "draw everything that you saw earlier as precisely as possible" (this was designed to avoid using labeling words such as "shape" or "rectangle" that might explicitly evoke the concept of rectangularity).

Analysis. We measured the ratio of the drawn rectangle by dividing the average length of the long sides by the average of short sides. The degree of amplification was the log of the ratio between the response ratio and the ratio of the $1.2 \mathrm{x}$-exaggerated rectangle in the memorandum. Thus, the number was zero if there were no bias in any direction; positive and negative values if the response rectangle was more and less extreme than the one they had to remember respectively. The log scale means that $2 \mathrm{x}$ and $0.5 \mathrm{x}$ become the same magnitude with a different sign $(0.69$ and -0.69 respectively in this example). A one-sample two-sided t-test was performed to demonstrate statistical differences.

\section{Results}

All participants drew skinnier rectangles ( 9 out of 9, Figure 1C., $\mathrm{t}(8)=4.156, p=0.003$, Cohen's d of 1.39) than the original memoranda. This distortion was not toward the prototypical rectangular ratio as the rectangle in the memoranda was already more rectangular than the prototype. Thus, we find distinctiveness exaggeration - the rectangle is remembered as more rectangular than it was.

\section{EXPERIMENT II}

Our stimulus in Experiment I consisted not only of the rectangle but also of a square shape that served as a distractor. Thus, in Experiment II we examined whether the effect in Experiment I was driven by the presence of the square. In particular, we asked whether the ratio of the rectangle would still be exaggerated even without the square. In addition, we asked whether this distinctiveness exaggeration effect was a result of initial encoding, or whether it changed in memory. To answer this, we tested at different time delays, ranging from 2 minutes to 7 days.

\section{Methods}

Participants. We predicted that the exaggeration effect will be substantially smaller when there was only one object to remember. Thus, we set the effect size at 1.0 (a minimum number of participants of 16 , alpha $=0.05$, power $=0.80)$. Twenty-one healthy volunteers $(14$ female, mean age of $20.9 \pm 0.6$ S.E.M.) from the University of California San Diego (UCSD) community participated in the experiment. All procedures were approved by the UCSD Institutional Research Board. All participants reported normal or corrected-to-normal vision without color-blindness and provided written informed consent. Participants were naïve to the purpose of the study and 
received course credit for their time. Four subjects failed to show up to both study sessions and excluded from the experiment.

Stimuli. We repeated the stimulus preparation step from Experiment I; in particular, we acquired each individual's prototypical rectangles a few weeks before the memory experiment and created exaggerated rectangles for each participant. In Experiment II, however, we now presented these exaggerated rectangles without a square. In particular, the rectangle was now in the middle of the page and slightly tilted so that participants needed to remember not just the rectangle's aspect ratio but also the orientation of the rectangle as an additional feature.

Procedure. The same instructions were given to the participants. After encoding the memoranda, participants then performed a distractor task (passive listening to a story). Unbeknownst to the subjects, they then had to reproduce the rectangle at both 2 and 7 minutes after initial memory acquisition (Figure 2). We also repeated the test after 7 days to measure the effect of a longer delay.

Analysis. A one-sample two-sided t-test was performed to demonstrate statistical differences.

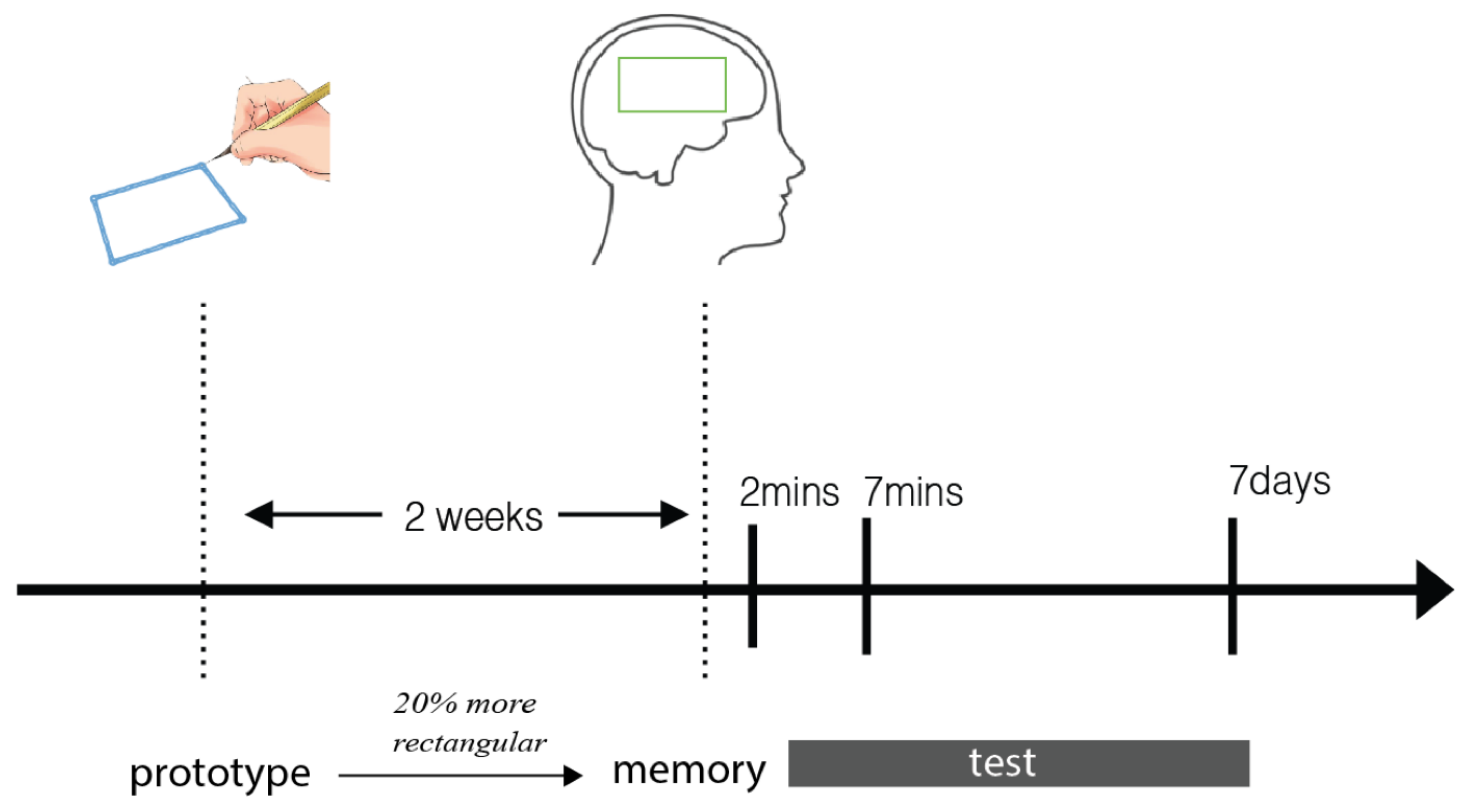

Figure 2. Timeline in Experiment II. Each subject first drew a rectangle (prototype). The memoranda were then individually constructed to be $20 \%$ more rectangular than each participant's prototypical rectangle. These memoranda were shown to participants, who then had to draw what they remembered at 2 minutes, 7 minutes and 7 days after the exposure.

\section{Results}

Seventeen participants completed Experiment II. The effects were still present in the absence of a square, but the effect arose only after a delay that was sufficient to prevent nearly 
perfect memory. In particular, we found 8/17, 12/17 and 9/17 participants drew an exaggerated rectangle at 2 minutes, 7 minutes and 7 days respectively. There was initially no significant exaggeration of the ratio at 2 minutes delay $(\mathrm{t}(16)=0.15, \mathrm{p}>0.05)$ but then became significantly more exaggerated at 7 minutes delays $(\mathrm{t}(16)=2.93, \mathrm{p}=0.01, \mathrm{~d}=0.71)$. We did not find an exaggeration of memory at 7 days delay $(t(16)=0.47, p>0.05)$. However, planned $t$-tests showed a significant increment in the exaggeration effect between 2-min versus 7-min delay $(t(16)=2.83$, $\mathrm{p}=0.012, \mathrm{~d}=0.69)$, and this difference survived a Bonferroni correction.

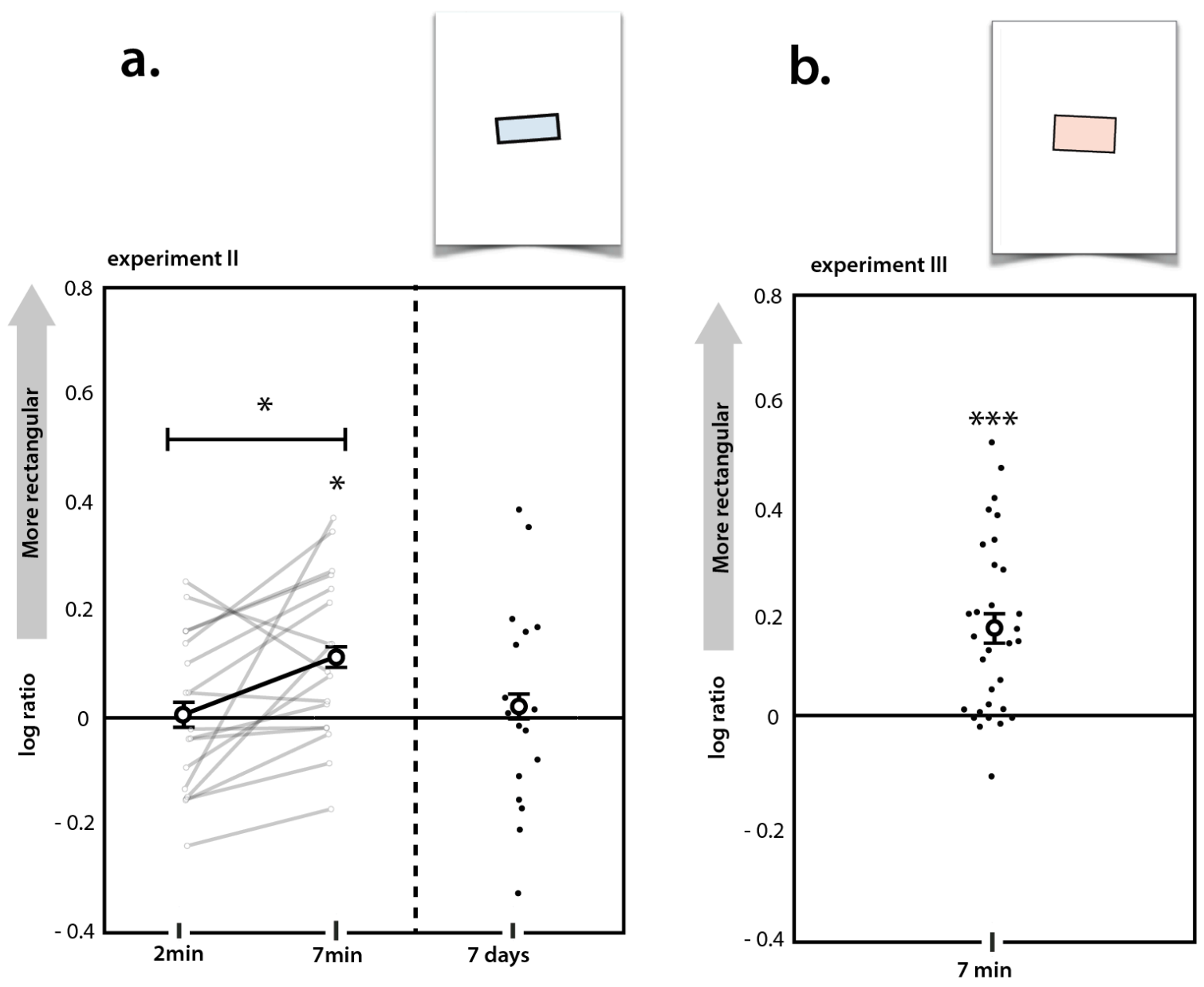

Figure 3. (a) Results from Experiment II in which we removed the square to demonstrate that the effect was not solely driven by the presence of the square. There were significant exaggerations of aspect ratio after 7-minutes, though not when tested immediately (at 2 minutes). The exaggeration at 7-minutes was also significantly larger than at the 2-minute delay. (b) Results from Experiment III. The participants were shown and had to remember a 20\% less rectangular shape than the ones they drew initially (their prototype). Participants continued to exaggerate the aspect ratio (rectangularity). 
The fact that the effect grew slightly larger with longer delays within the first 10 minutes implies that the process of distinctiveness exaggeration continues after initial memory acquisition. The fact that the amplification effect was not present after 7 days is not surprising because we predicted that as the memory became very imprecise, participants would likely revert to their prototypical rectangle (i.e., for those subjects who no longer remember the specific rectangle that we gave, they will draw something close to their prototypical rectangle). Thus, the amplification effect seems to grow stronger as memory became less precise but then disappears when the memory is too weak.

\section{EXPERIMENT III}

The results from first two experiments demonstrated that the reproduced rectangles were more extreme than the rectangle that they had to remember, even if that initial rectangle was already 1.2 times as rectangular as their prototype rectangle. One possibility is that participants compared the to-be-remembered rectangle to their own prototypical rectangle (i.e., "skinner" than a usual rectangle). In this case, we would expect that the distortion would flip if participants are asked to remember a rectangle that is more "square" than their prototypical rectangle. However, another possibility is that the difference between the length of long and short sides was exaggerated. If this is the case, the rectangle would continue to be remembered as more rectangular even if it was more square than their prototype, since the prototype rectangle would not play a significant role in the encoding of the memory item.

\section{Methods}

Participants. We predicted that the effect size will be similar to the result from experiment 2 (i.e. serve as internal replication experiment, a minimum number of participants of $32, \mathrm{~d}=0.71$, alpha $=0.05$, power $=0.8)$. Thirty-two healthy volunteers ( 18 female, mean age of $20.9 \pm 0.3$ S.E.M. $)$ from the University of California San Diego (UCSD) community participated in the experiment. All procedures were approved by the UCSD Institutional Research Board. All participants reported normal or corrected-to-normal vision.

Stimuli. We repeated the stimulus preparation step from Experiment I and II (acquired individual's prototypical rectangles a few weeks before the memory experiment) but created a $20 \%$ less exaggerated rectangles for each participant $(0.8 \mathrm{x})$ as opposed to the more exaggerated rectangle $(1.2 \mathrm{x})$ of the previous experiments.

Procedure. The same instructions were given to the participants. Then, participants had to perform the distraction task (passive listening to a story). Memory was tested 7 minutes after memory encoding.

Analysis. A one-sample two-sided t-test was performed to demonstrate statistical differences. 


\section{Results}

Participants still drew more rectangular figures than the ones they were initially shown (26 out of 32 participants, $\mathrm{t}(31)=5.74, p<0.001, \mathrm{~d}=1.01$; Figure $3 \mathrm{~B}$ ) even when the memoranda were less rectangular than their own prototypical rectangles. This implies that the participants did not use their prototypical rectangle as the reference (i.e. neither implicitly or explicitly; e.g., they likely did not say to themselves "I remembered that it was a particularly skinny rectangle"). Thus, the most likely explanation is that the rectangles were encoded by considering the lengths of the longer and shorter sides separately, and the difference between the longer and shorter sides of the shape were contrasted and subsequently amplified.

\section{EXPERIMENT IV}

It is possible that the aspect ratio of a rectangle is a unique feature and this same principle does not apply more broadly. Thus in Experiment IV, we ask whether this phenomenon of exaggerating the distinction between features is a more general principle of memories by testing memory for relative size rather than shape. Participants needed to remember the size of two squares, and then draw them after a delay. We then asked if the distinction between them-- the size difference --was systematically exaggerated by participants in a drawing-based recall task.

\section{Methods}

Participants. The memory of the size might be more volatile than the ratio of the shape (e.g., the size of the objects in the real world can vary a lot depend on the viewing distance while the shape is relatively more stable). On contrary, having two contrasting objects might yield higher effect size as we found in experiment 1 . We expected the effect size to be between experiment 1 vs. experiment $2 \& 3$ (d at 1.2 with a minimum number of participants of 12 ). Twelve healthy volunteers ( 8 female, mean age of $21.1 \pm 0.4$ S.E.M.) from the University of California San Diego (UCSD) community participated in the experiment. All procedures were approved by the UCSD Institutional Research Board. All participants reported normal or corrected-to-normal vision.

Stimuli. Our memorandum consisted of a $1.4 \times 1.4$ inch and a $1.8 \times 1.8$ inch square (one in blue and one in red color, randomly assigned) on a standard $8.5 \times 11$ inch white piece of paper with black borders. The squares were placed in the upper left and lower right corners to avoid direct side-byside comparison.

Procedure. The same instructions were given to the participants, followed by memory encoding and then a distraction task. Memory was tested with a 1-minute delay.

Analysis. We computed the ratio between the surface area of the big and small square. We did this both for the original pair $(1.8 \times 1.8 / 1.4 \times 1.4=1.653)$ and for the ones drawn by each participant. The degree of size-difference amplification was quantified as the $\log$ of the ratio between the ratio of the original pair vs. the one the participants drew. A one-sample two-sided t-test was performed to test statistical differences. 


\section{Results}

When recalling the size of two squares, participants exaggerated the size difference between them by about $24 \%$ percent (10 out of 12 participants, $t(11)=3.019, p=0.012, d=0.87$ Figure 4). This shows that the exaggeration of the ratio of rectangles is not unique. In another situation where participants needed to encode the relationship between two items (the size of the squares as opposed to the length of the sides of the rectangle), this difference was once again exaggerated, suggesting that distinctiveness exaggeration is a more general phenomena, and that representations are in fact distorted in memory to amplify distinctive features.

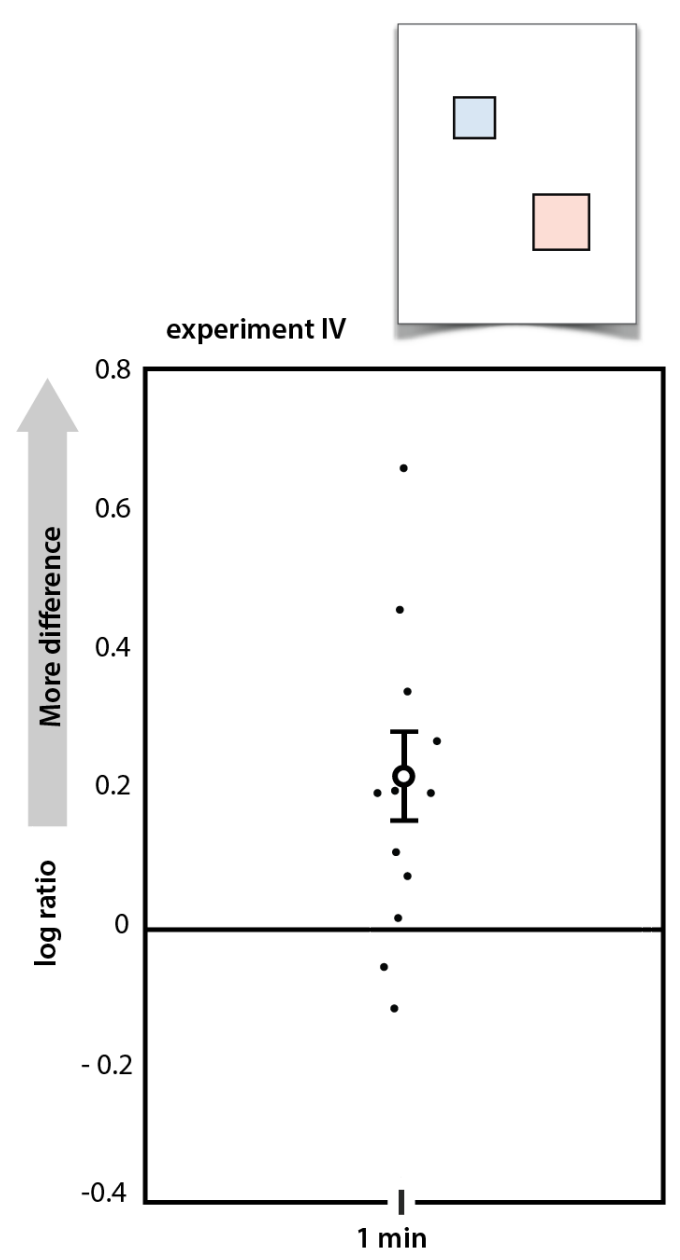

Figure 4. Results from Experiment IV. The subjects were shown two squares of different sizes. They showed an exaggeration of the size difference after a 1-minute delay. 


\section{DISCUSSION}

Many previous studies have shown that visual memory tends to be systematically distorted toward the gist or prototype (e.g., Huttenlocher et al., 1991, 2000). However, if one of the goals of our memory system is to avoid confusion, then when we need to remember one item or to distinguish two similar items, memories may instead become more distinct from other memories or from the category rather than more similar. The current experiments demonstrate that, at least in some circumstances, our visual memories are in fact systematically distorted such that the unique (diagnostic) features of an item are amplified. Additionally, this amplification grows stronger with longer delays, suggesting that the process of distinctiveness exaggeration continues after initial memory acquisition. Importantly, these results show that caricature effects (Lee et al., 2000; Mauro \& Kubovy, 1992) are not solely due to the way stimuli are matched to otherwise veridical memories. Instead, memories themselves are sometimes distorted to exaggerate their distinctiveness.

\section{Why exaggeration?}

All memory must be a compromise between the opposing tendencies of attraction to the category center, to make the best use of gist information, and repulsion from the category center (exaggeration), to keep items distinct from each other. Neither overgeneralization nor total focus on learning a specific exemplar can result in optimal learning (Xu \& Südhof, 2013). The use of these different strategies for encoding memories therefore likely differs by circumstance. In some circumstances, where we wish to recognize one object in particular as distinct from other similar objects (i.e., specificity is the goal), blending that particular object with similar ones will not allow us to achieve our goal, even if it minimizes our average error for remembering many items (e.g., Huttenlocher et al. 2001). Instead, to maintain maximum specificity for a particular item, memory should be biased in the opposite direction - amplifying the diagnostic features of an object or repulsing it from the category or gist. This should result in a caricature version of that object instead of a version that is blended together with the other similar objects.

Here we show this repulsion bias occurs in visual memory by using a recall task, thus ensuring that it is not the recognition matching process that benefits from such caricature but actually the underlying representation that is affected. In addition, we show that this occurs in memory, not perception since it increases over the course of the first several minutes. We also show this is a form of distinctiveness exaggeration rather than purely a relational encoding effect (e.g., repulsion bias) because it occurs even when only one stimulus must be remembered. However, this effect is closely related to previous work which shows repulsion biases when two closely related items are perceived. For example, when a participants look at two intersecting lines with similar orientations or two similar motion directions (either simultaneously or sequentially), they often perceive the angle between them as wider than it is (e.g., O'Toole \& Wenderoth, 1977; 
Schwartz, Hsu, \& Dayan, 2007; Marshak \& Sekuler, 1979; Rauber \& Treue, 1998) as well as other visual features e.g., spatial frequency (Klein, Stromeyer, \& Ganz, 1974), curve line (Gibson, 1933) and oval shapes (Sweeny, Grabowecky, \& Suzuki, 2011). Recent studies demonstrated a close relationship between the perceptual repulsion bias and perceptual discriminability (Wei \& Stocker, $2015,2017)$. This is a perceptual analog of the distinctiveness exaggeration effect we report here, and of the repulsion that results from this distinctiveness exaggeration when encoding two stimuli in memory (e.g., Experiment I \& IV). Together, our data suggest that while attraction toward the center of the category may be the most common result -- when people need to remember many items, this helps promotes generalization of information to inform many items at once -exaggeration or repulsion away from the center of the category may promote specificity in circumstances where one or few items need to be remembered as distinct from a category or set of items.

\section{CONCLUSION}

Memory is a constructive and adaptive process. Instead of maintaining information as accurately as possible, the content of our memory takes into account the relation between items, our pre-existing knowledge, and our current reference frame. By studying visual memory for simple shapes and sizes, here we demonstrate that memory is not always biased toward the prototype or center of a category. On the contrary, we show that certain diagnostic features are amplified in memory, presumably to make these items more distinctiveness from the rest of the items in that category or from similar items that we have encountered. This trajectory of distinctiveness exaggeration even continues after initial encoding. Thus, we propose that amplification of distinctive features will occur when two or more things are distinct but potentially confusable, and we are tasked with remembering one of them in particular. This may help our brains reduce confusability between items when our goal is specificity, rather than remembering many items as accurately as possible. It is a curious thought that when you walk around acquiring new memories, hours afterward, your memories are not a replica, but are instead a caricatured version of the world. 


\section{REFERENCES}

Alvarez, G. A. (2011). Representing multiple objects as an ensemble enhances visual cognition. Trends in Cognitive Sciences, 15(3), 122-131.

Bartlett, F. C. (1932). Some Experiments on the Reproduction of Folk-Stories. Folklore, 31(1), $30-47$.

Brady, T. F., \& Alvarez, G. A. (2011). Hierarchical encoding in visual working memory: ensemble statistics bias memory for individual items. Psychological Science, 22(3), 384392.

Dubé, C., Zhou, F., Kahana, M. J., \& Sekuler, R. (2014). Similarity-based distortion of visual short-term memory is due to perceptual averaging. Vision Research, 96, 8-16.

Freyd, J. J., \& Johnson, J. Q. (1987). Probing the time course of representational momentum. Journal of Experimental Psychology. Learning, Memory, and Cognition, 13(2), 259-268.

Gibson, J. J. (1933). Adaptation, after-effect and contrast in the perception of curved lines. Journal of Experimental Psychology, 16(1), 1.

Hemmer, P., \& Steyvers, M. (2009). A Bayesian account of reconstructive memory. Topics in Cognitive Science, 1(1), 189-202.

Huang, J., \& Sekuler, R. (2010). Distortions in recall from visual memory: two classes of attractors at work. Journal of Vision, 10(2), 24.1-27.

Hubbard, T. L., Hutchison, J. L., \& Courtney, J. R. (2010). Boundary extension: findings and theories. Quarterly Journal of Experimental Psychology , 63(8), 1467-1494.

Huttenlocher, J., Hedges, L. V., \& Duncan, S. (1991). Categories and particulars: prototype effects in estimating spatial location. Psychological Review, 98(3), 352-376.

Huttenlocher, J., Hedges, L. V., \& Vevea, J. L. (2000). Why do categories affect stimulus judgment? Journal of Experimental Psychology. General, 129(2), 220-241.

Intraub, H., \& Richardson, M. (1989). Wide-angle memories of close-up scenes. Journal of Experimental Psychology. Learning, Memory, and Cognition, 15(2), 179-187.

Klein, S., Stromeyer, C. F., 3rd, \& Ganz, L. (1974). The simultaneous spatial frequency shift: a dissociation between the detection and perception of gratings. Vision Research, 14(12), $1421-1432$. 
Lee, K., Byatt, G., \& Rhodes, G. (2000). Caricature effects, distinctiveness, and identification: testing the face-space framework. Psychological Science, 11(5), 379-385.

Marshak, W., \& Sekuler, R. (1979). Mutual repulsion between moving visual targets. Science, 205(4413), 1399-1401.

Mauro, R., \& Kubovy, M. (1992). Caricature and face recognition. Memory \& Cognition, 20(4), $433-440$.

O'Toole, B., \& Wenderoth, P. (1977). The tilt illusion: repulsion and attraction effects in the oblique meridian. Vision Research, 17(3), 367-374.

Rauber, H. J., \& Treue, S. (1998). Reference repulsion when judging the direction of visual motion. Perception, 27(4), 393-402.

Schacter, D. L., Guerin, S. A., \& St. Jacques, P. L. (2011). Memory distortion: an adaptive perspective. Trends in Cognitive Sciences, 15(10), 467-474.

Schwartz, O., Hsu, A., \& Dayan, P. (2007). Space and time in visual context. Nature Reviews. Neuroscience, 8, 522 .

Spencer, J. P., \& Hund, A. M. (2002). Prototypes and particulars: geometric and experiencedependent spatial categories. Journal of Experimental Psychology. General, 131(1), 16-37.

Sweeny, T. D., Grabowecky, M., \& Suzuki, S. (2011). Simultaneous shape repulsion and global assimilation in the perception of aspect ratio. Journal of Vision, 11(1), 16.

Tanaka, J. W. (1996). Caricature Recognition in a Neural Network. Visual Cognition, 3(4), 305324.

Wei, X. X., \& Stocker, A. A. (2015). A Bayesian observer model constrained by efficient coding can explain 'anti-Bayesian' percepts. Nature neuroscience, 18(10), 1509.

Wei, X. X., \& Stocker, A. A. (2017). Lawful relation between perceptual bias and discriminability. Proceedings of the National Academy of Sciences, 114(38), 10244-10249.

Xu, W., \& Südhof, T. C. (2013). A neural circuit for memory specificity and generalization. Science, 339(6125), 1290-1295.

Yassa, M. A., \& Stark, C. E. L. (2011). Pattern separation in the hippocampus. Trends in Neurosciences, 34(10), 515-525. 